\title{
LETTERS
}

\section{Post-test Probability According to Prevalence}

\author{
Benjamin T. Galen, MD \\ Resident in Internal Medicine, Yale University School of Medicine, New Haven, CT, USA.
}

J Gen Intern Med 26(10):1090

DOI: $10.1007 / \mathrm{s} 11606-011-1787-5$

( ) Society of General Internal Medicine 2011

To the Editor:- The impact of disease prevalence on post-test disease probability cannot be understated and Agoritsas, et al. demonstrate clearly that their survey population tends towards overestimation of disease probability given a positive test result: the majority of respondents in each category chose the 95-99.9\% option. The authors assert that incorporating disease prevalence (or prior probability) is not intuitive to clinicians and they propose as evidence the observation that physicians who were not provided with prevalence data in their study still "guessed" at post-test probability. However, it is unclear to the reader if these subjects even had the option in their survey to reply "cannot be determined," since Table 1 indicates that $100 \%$ of these respondents did in fact select a post-test probability. Conclusions drawn from this arm of the study are therefore limited.

This trial underscores the limitations of teaching arithmeticbased Bayesian calculations to medical trainees, since such statistical calculations are clinically cumbersome to implement if only because the terminology used to evaluate laboratory tests is not intuitive. The concepts are, however, quite intuitive and it has been shown that $95 \%$ of physicians who do take into consideration test characteristics in interpreting results do so in a "informal direct method"1. Rather than thinking in terms of ratios, odds, or nomograms, it is ideal to know which tests are good for ruling out and which are good for ruling in and when to use them. Leaving the mathematical heavy lifting to those who develop evidence-based guidelines in diagnostics permits clinicians to utilize Bayesian principles at the bedside without their knowing it. A good example is the extensive work done to optimize $\mathrm{d}$-dimer testing and imaging in evaluating a patient for $\mathrm{PE}^{2,3}$. The trial by Agoritsas et al. reminds us that clinicians do need this structure in order to properly use and interpret diagnostic tests.

Corresponding Author: Benjamin T. Galen, MD; Resident in Internal Medicine, Yale University School of Medicine, 95 Avon Street, New Haven, CT 065111, USA (e-mail: Benjamin.galen@yale. edu).

\section{REFERENCES}

1. Reid MC, Lane DA, Feinstein AR. Academic calculations versus clinical judgments: practicing physicians' use of quantitative measures of test accuracy. Am J Med. 1998;104(4):374-80.

2. Wells PS, Owen C, Doucette S, Fergusson D, Tran H. Does this patient have deep vein thrombosis? JAMA. 2006;295(2):199-207.

3. British Thoracic Society Standards of Care Committee Pulmonary Embolism Guideline Development Group. British Thoracic Society guidelines for the management of suspected acute pulmonary embolism. Thorax 2003;58(6):470-484 